\title{
Synchronization between Two Discrete-Time Networks with Mutual Couplings
}

\author{
Meng Xiao, Weigang Sun, and Fangyue Chen \\ Institute of Applied Mathematics and Engineering Computations, Hangzhou Dianzi University, Hangzhou 310018, China \\ Correspondence should be addressed to Weigang Sun; wgsun@hdu.edu.cn
}

Received 22 September 2013; Accepted 4 November 2013

Academic Editor: Jianquan Lu

Copyright (C) 2013 Meng Xiao et al. This is an open access article distributed under the Creative Commons Attribution License, which permits unrestricted use, distribution, and reproduction in any medium, provided the original work is properly cited.

\begin{abstract}
We investigate synchronization between two discrete-time networks with mutual couplings, including inner synchronization inside each network and outer synchronization between two networks. We then obtain a synchronized criterion for the inner synchronization inside each network by the method of linear matrix inequality and derive a relationship between the inner and outer synchronization. Finally, we show numerical examples to verify our theoretical analysis and discuss the effect of coupling strengths, node dynamics, and topological structures on the inner and outer synchronization. Compared to the inner synchronization inside each network, the outer synchronization between two networks is difficult to achieve.
\end{abstract}

\section{Introduction}

Network synchronization, as a collective behavior existing inside a network, has been widely studied since the birth of small-world and scale-free networks [1-3]. The main focus is to investigate the interplay between the complexity in the overall topology and the local dynamics of the coupled nodes [4-6]. The topological structures may be globally connected, random, small-world, and scale-free. There are many applications using the synchronization of networks [7], for instance, secure communication and multirobot coordination control. Apart from the complete synchronization appearing inside a network, there are some other types of synchronization, such as phase synchronization, generalized synchronization, lag synchronization, and cluster synchronization [8-12].

Generally, we refer to synchronization happening between two networks as outer synchronization [13], which is distinguished from inner synchronization inside a network. Compared to the inner synchronization, outer synchronization of two networks is more complex, which involves more system parameters. In 2007, Li et al. first proposed the concept of outer synchronization and applied the open-plusclosed-loop method to realize the outer synchronization between two networks with identical topologies [13]. Shortly later, using the adaptive control method, Tang et al. achieved the outer synchronization between two networks with different topological structures [14]. In [15], Wu et al. studied the generalized outer synchronization between two networks with different dimensions of node dynamics. In addition, there are many works on the outer synchronization, that is, introducing the noise, time delay, fractional order node dynamics, and unknown parameters [16-21].

In the above-mentioned works on the outer synchronization, the researchers usually applied the control methods to realize the outer synchronization and did not study the inner synchronization inside a network. In reality, the mutual coupling forms between two networks are colorful; for instance, Wu et al. investigated the outer synchronization between two networks with two active forms nonlinear signals and reciprocity [22]; however, these two coupling forms do not make the outer synchronization happen. In addition, the inner synchronization inside each network was not considered. In [23], Sorrentino and Ott provided a method to study the inner synchronization of two groups. The problem of collective behaviors inside a network and between two networks is of broad interest. For example, in subway systems, when the trains reach the platform, the outer and inner doors simultaneously open or close, showing that both inner and outer synchronization happen [24]. It is also found 
that present studies on the synchronization between two networks with various couplings are much less, then studying the effect of various couplings on the synchronization is interesting and meaningful.

Inspired by the above discussions, we study synchronization between two discrete-time networks with mutual couplings, including inner synchronization inside each network and outer synchronization between them. By the Lyapunov stability theory and linear matrix inequality, we obtain a synchronous theorem on the inner synchronization inside each network and a relationship between the inner and outer synchronization. Numerical simulations show that the inner synchronization is easier to achieve than the outer synchronization. In addition, given the mutual coupling matrices and appropriate node dynamics, we can adjust coupling strengths to realize the inner and outer synchronization simultaneously. In Section 2, network models and synchronization analysis are presented, and numerical examples are shown in Section 3. Finally, the discussions are included in the last section.

\section{Model Presentation and Synchronization Analysis}

In this paper, we investigate the synchronization between two discrete-time networks with mutual couplings. The dynamical equations are described as follows:

$$
\begin{array}{r}
x_{i}(t+1)=f\left(x_{i}(t)\right)+m_{x} \sum_{j=1}^{N} a_{i j}\left(y_{j}(t)-x_{j}(t)\right), \\
i=1,2, \ldots, N, \\
y_{i}(t+1)=g\left(y_{i}(t)\right)+m_{y} \sum_{j=1}^{N} b_{i j}\left(x_{j}(t)-y_{j}(t)\right), \\
i=1,2, \ldots, N,
\end{array}
$$

where the node dynamical equations are $x_{i}(t+1)=f\left(x_{i}(t)\right)$ and $y_{i}(t+1)=g\left(y_{i}(t)\right), i=1, \ldots, N \cdot f(\cdot): R^{n} \rightarrow R^{n}$ and $g(\cdot): R^{n} \rightarrow R^{n}$ are continuously differential functions. $x_{i}\left(y_{i}\right)$ is an $n$-dimensional state vector. $N$ is the number of network nodes. $m_{x}$ and $m_{y}$ are the coupling strengths. $A=\left(a_{i j}\right)_{N \times N}$ and $B=\left(b_{i j}\right)_{N \times N}$ represent the mutual coupling matrices between these two networks, whose entries $a_{i j}$ denote the intensity from $i$ in network $X$ to $j$ in network $Y$; analogously, the entries of $B$ are the same defined as $A$.

Let us now consider the possibility whether the individual networks achieve inner synchronization; that is, $\lim _{t \rightarrow+\infty}\left\|x_{i}(t)-x_{s}(t)\right\|=0$ and $\lim _{t \rightarrow+\infty}\left\|y_{i}(t)-y_{s}(t)\right\|=$ $0, i=1 \ldots, N$, where $\|\cdot\|$ denotes the Euclidean norm of a vector. If there exist such synchronous states, satisfying

$$
\begin{aligned}
& \sum_{j=1}^{N} a_{i j}=\gamma_{x}, \quad \forall i \in X, \\
& \sum_{j=1}^{N} b_{i j}=\gamma_{y}, \quad \forall i \in Y,
\end{aligned}
$$

without loss of generality, we set $\gamma_{x}=\gamma_{y}=1$.
Thus the synchronized state equations are

$$
\begin{aligned}
& x_{s}(t+1)=f\left(x_{s}(t)\right)+m_{x}\left(y_{s}(t)-x_{s}(t)\right), \\
& y_{s}(t+1)=g\left(y_{s}(t)\right)+m_{y}\left(x_{s}(t)-y_{s}(t)\right) .
\end{aligned}
$$

Linearizing the synchronous states around $x_{s}$ and $y_{s}$, we obtain

$$
\begin{aligned}
\delta x_{i}(t+1)= & D f\left(x_{s}(t)\right) \delta x_{i}(t) \\
& +m_{x} \sum_{j=1}^{N} a_{i j}\left(\delta y_{j}(t)-\delta x_{j}(t)\right), \quad i=1,2, \ldots, N, \\
\delta y_{i}(t+1)= & D g\left(y_{s}(t)\right) \delta y_{i}(t) \\
& +m_{y} \sum_{j=1}^{N} b_{i j}\left(\delta x_{j}(t)-\delta y_{j}(t)\right), \quad i=1,2, \ldots, N,
\end{aligned}
$$

where $\delta x_{i}(t)=x_{i}(t)-x_{s}(t), \delta y_{i}(t)=y_{i}(t)-y_{s}(t), D f\left(x_{s}(t)\right)$, and $D g\left(y_{s}(t)\right)$ are the Jacobians of $f(x(t)), g(y(t))$ at $x_{s}$ and $y_{s}$, respectively. Assume $A=B$ and let $\delta x(t)=$ $\in R^{n \times N}$ and $\delta y(t)=\left[\delta y_{1}(t), \ldots, \delta y_{N}(t)\right] \in R^{n \times N}$. Then (4) is rewritten as

$$
\begin{aligned}
& \delta x(t+1)=D f\left(x_{s}(t)\right) \delta x(t)+m_{x}(\delta y(t)-\delta x(t)) A^{T}, \\
& \delta y(t+1)=D g\left(y_{s}(t)\right) \delta y(t)+m_{y}(\delta x(t)-\delta y(t)) A^{T} .
\end{aligned}
$$

Further, let $\delta(t)=\left[\begin{array}{l}\delta x(t) \\ \delta y(t)\end{array}\right] \in R^{2 n \times N}$; then (5) reads

$$
\delta(t+1)=\mathscr{D} \delta(t)+\mathscr{W} \delta(t) A^{T}
$$

where $\mathscr{D}=\left[\begin{array}{cc}D f\left(x_{s}(t)\right) & 0 \\ 0 & D g\left(y_{s}(t)\right)\end{array}\right]$ and $\mathscr{W}=\left[\begin{array}{cc}-m_{x} I_{n} & m_{x} I_{n} \\ m_{y} I_{n} & -m_{y} I_{n}\end{array}\right]$, where $I_{n}$ is an identity matrix of size $n$ and $A^{T}$ denotes the transpose of $A$. Generally, the coupling matrix can be decomposed into $A^{T}=\Phi J \Phi^{-1}$, where $J$ is the Jordan canonical form with complex eigenvalues $\lambda \in C$ and $\Phi$ contains the corresponding eigenvectors $\phi$. Denote $\eta(t)=\delta(t) \Phi$; we obtain

$$
\eta(t+1)=\mathscr{D} \eta(t)+\mathscr{W} \eta(t) J
$$

where $J$ is a block diagonal matrix; that is,

$$
J=\left[\begin{array}{lll}
J_{1} & & \\
& \ddots & \\
& & J_{h}
\end{array}\right],
$$

and $J_{k}$ is the block corresponding to the $N_{k}$ multiple eigenvalue $\lambda_{k}$ of $A^{T}$; that is,

$$
J_{k}=\left[\begin{array}{ccccc}
\lambda_{k} & 1 & 0 & \cdots & 0 \\
0 & \lambda_{k} & 1 & \cdots & 0 \\
\vdots & \vdots & \ddots & \ddots & \vdots \\
0 & 0 & \cdots & \lambda_{k} & 1 \\
0 & 0 & \cdots & 0 & \lambda_{k}
\end{array}\right]
$$


Let $\eta(t)=\left[\eta_{1}(t), \eta_{2}(t), \ldots, \eta_{h}(t)\right]$ and $\eta_{k}(t)=\left[\eta_{k, 1}(t), \eta_{k, 2}(t)\right.$, $\left.\ldots, \eta_{k, N_{k}}(t)\right]$. We can rewrite (7) in a component form as

$$
\begin{gathered}
\eta_{k, 1}(t+1)=\left(\mathscr{D}+\lambda_{k} \mathscr{W}\right) \eta_{k, 1}(t), \\
\eta_{k, p+1}(t+1)=\left(\mathscr{D}+\lambda_{k} \mathscr{W}\right) \eta_{k, p+1}(t)+\mathscr{W} \eta_{k, p}(t), \\
1 \leq p \leq N_{k}-1,
\end{gathered}
$$

where $k=1,2, \ldots, h$.

Firstly, we study the system of (10). Let $\eta_{k, 1}(t)=\mu_{k, 1}(t)+$ $j v_{k, 1}(t), \lambda_{k}=\alpha_{k}+j \beta_{k}$, where $\alpha_{k}, \beta_{k} \in R, \mu_{k, 1}, v_{k, 1} \in R^{2 n}, j$ is and the imaginary unit. Then (10) reads

$$
\begin{aligned}
& \mu_{k, 1}(t+1)=\left(\mathscr{D}+\alpha_{k} \mathscr{W}\right) \mu_{k, 1}(t)-\beta_{k} \mathscr{W} v_{k, 1}(t), \\
& v_{k, 1}(t+1)=\left(\mathscr{D}+\alpha_{k} \mathscr{W}\right) v_{k, 1}(t)+\beta_{k} \mathscr{W} \mu_{k, 1}(t) .
\end{aligned}
$$

Construct the Lyapunov function as

$$
V(t)=\mu_{k, 1}^{T}(t) \mu_{k, 1}(t)+v_{k, 1}^{T}(t) v_{k, 1}(t) .
$$

Then,

$$
\begin{aligned}
\Delta V(t)= & V(t+1)-V(t) \\
= & \mu_{k, 1}^{T}(t+1) \mu_{k, 1}(t+1)+v_{k, 1}^{T}(t+1) v_{k, 1}(t+1) \\
& -\mu_{k, 1}^{T}(t) \mu_{k, 1}(t)-v_{k, 1}^{T}(t) v_{k, 1}(t) \\
= & {\left[\begin{array}{c}
\mu_{k, 1}(t) \\
v_{k, 1}(t)
\end{array}\right]^{T} M_{k}\left[\begin{array}{c}
\mu_{k, 1}(t) \\
v_{k, 1}(t)
\end{array}\right], }
\end{aligned}
$$

where $M_{k}=P_{k}^{T} P_{k}-\operatorname{diag}\left\{I_{2 n}, I_{2 n}\right\}$ with $P_{k}=\left[\begin{array}{ccc}\mathscr{D}+\alpha_{k} \mathscr{W} & -\beta_{k} \mathscr{W} \\ \beta_{k} \mathscr{W} & \mathscr{D}+\alpha_{k} \mathscr{W}\end{array}\right]$, $k=1,2, \ldots, h$. If $M_{k}<0, k=1,2, \ldots, h$, that is, these matrices are negative definite, then the zero solution of (10) is asymptotically stable.

Secondly, we study the stability of (11). Let $\eta_{k, p+1}(t)=$ $\mu_{k, p+1}(t)+j v_{k, p+1}(t) ;$ then

$$
\begin{aligned}
& \mu_{k, p+1}(t+1)=\left(\mathscr{D}+\alpha_{k} \mathscr{W}\right) \mu_{k, p+1}(t) \\
&-\beta_{k} \mathscr{W} v_{k, p+1}(t)+\mathscr{W} \mu_{k, p}(t), \\
& 1 \leq p \leq N_{k}-1, \\
& v_{k, p+1}(t+1)=\left(\mathscr{D}+\alpha_{k} \mathscr{W}\right) v_{k, p+1}(t) \\
&+\beta_{k} \mathscr{W} \mu_{k, p+1}(t)+\mathscr{W} v_{k, p}(t), \\
& 1 \leq p \leq N_{k}-1 .
\end{aligned}
$$

Choose the Lyapunov function as

$$
\bar{V}(t)=\mu_{k, p+1}^{T}(t) \mu_{k, p+1}(t)+v_{k, p+1}^{T}(t) v_{k, p+1}(t) .
$$

Then we obtain

$$
\begin{aligned}
\Delta \bar{V}(t) & =\bar{V}(t+1)-\bar{V}(t) \\
& =\left[\begin{array}{c}
u_{k, p}(t) \\
v_{k, p}(t) \\
u_{k, p+1}(t) \\
v_{k, p+1}(t)
\end{array}\right]^{T} L_{k}\left[\begin{array}{c}
u_{k, p}(t) \\
v_{k, p}(t) \\
u_{k, p+1}(t) \\
v_{k, p+1}(t)
\end{array}\right],
\end{aligned}
$$

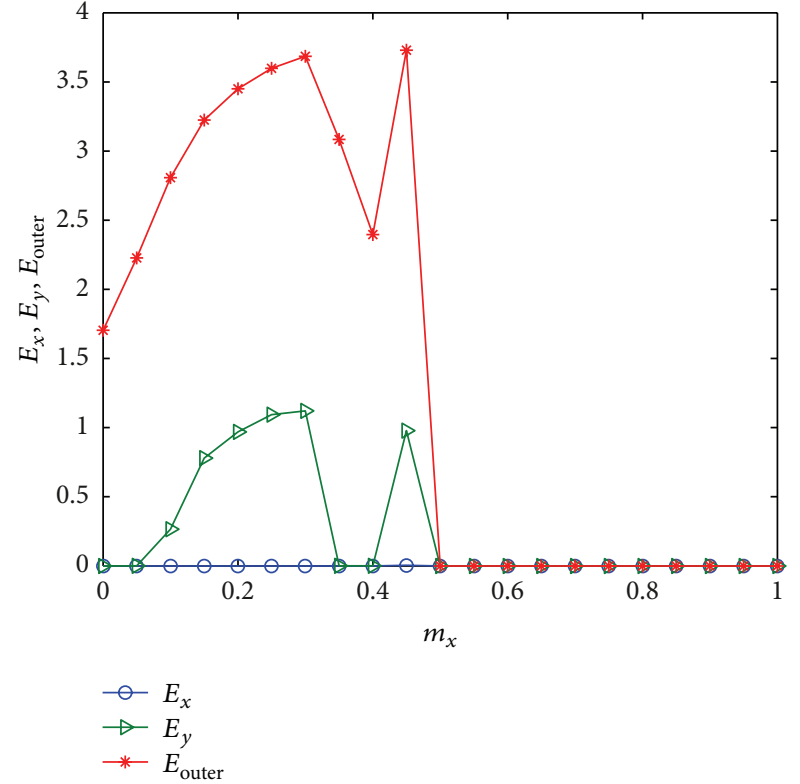

FIgure 1: The panels exhibit $E_{x}, E_{y}$, and $E_{\text {outer }}$ at $t=400$ with regard to $m_{x}$ for $N=10$ and $m_{y}=0.2$. The bottom one shows that the inner synchronization inside network $X$ is easily achieved. When $m_{x} \geq 0.5$, the inner and outer synchronization simultaneously appear.

where $L_{k}=Q_{k}^{T} Q_{k}-\operatorname{diag}\left\{0,0, \mathscr{W}^{T} \mathscr{W}+I_{2 n}, \mathscr{W}^{T} \mathscr{W}+I_{2 n}\right\}$ with

$$
Q_{k}=\left[\begin{array}{cccc}
0 & 0 & \mathscr{W} & 0 \\
0 & 0 & 0 & \mathscr{W} \\
\mathscr{W} & 0 & \mathscr{D}+\alpha_{k} \mathscr{W} & -\beta_{k} \mathscr{W} \\
0 & \mathscr{W} & \beta_{k} \mathscr{W} & \mathscr{D}+\alpha_{k} \mathscr{W}
\end{array}\right]
$$

If $L_{k}<0, k=1,2, \ldots, h$, then the zero solution of (11) is asymptotically stable. Hence we obtain a synchronized theorem for networks (1).

Theorem 1. Consider network systems (1). Assume the mutual coupling matrices $A=B$. Let $\lambda_{k}=\alpha_{k}+j \beta_{k}$ be the eigenvalues of $A$, where $\alpha_{k}, \beta_{k} \in R$. If these matrices $M_{k}, L_{k}<$ $0, k=1,2, \ldots, h$, then the networks (1) will achieve inner synchronization inside each network.

Remark 2. Note that Theorem 1 only gives a feasibility of the inner synchronization inside each network. When the inner synchronization inside networks $X$ and $Y$ happens, and the synchronized states $\left\|x_{s}(t)-y_{s}(t)\right\| \rightarrow 0$ for a large time, then the outer synchronization between networks $X$ and $Y$ will be achieved.

\section{Numerical Examples}

In this section, we will give some examples to illustrate our theoretical results obtained in the previous section. We mainly investigate the effect of coupling strengths, node dynamics, and mutual coupling forms on the inner and outer 


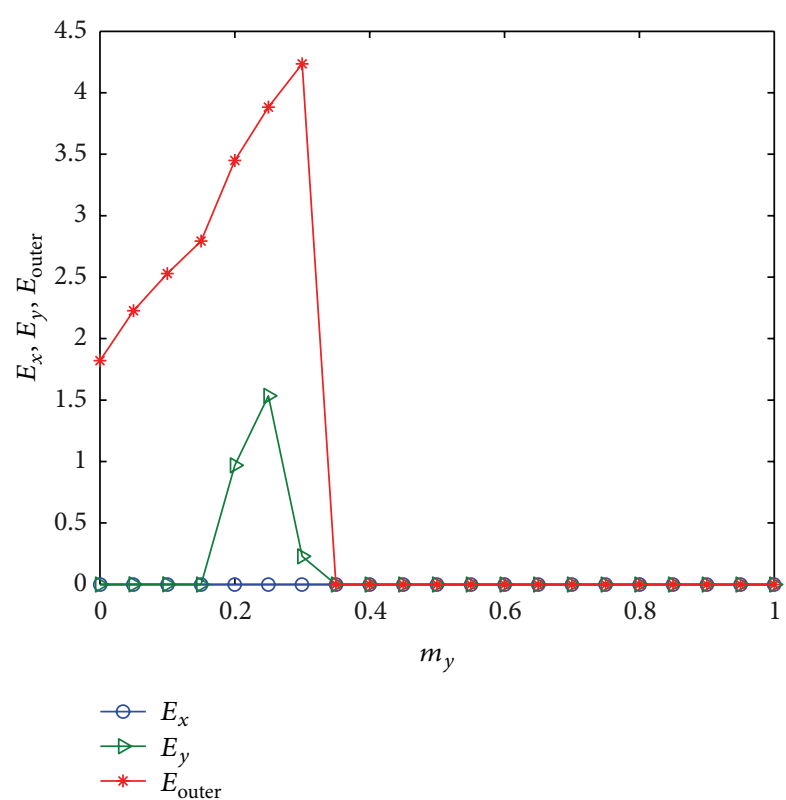

FIgURE 2: The plots show $E_{x}, E_{y}$, and $E_{\text {outer }}$ at $t=400$ on $m_{y}$ for $N=10$ and $m_{x}=0.3$. When $m_{y} \geq 0.35$, the inner and outer synchronization simultaneously happen.

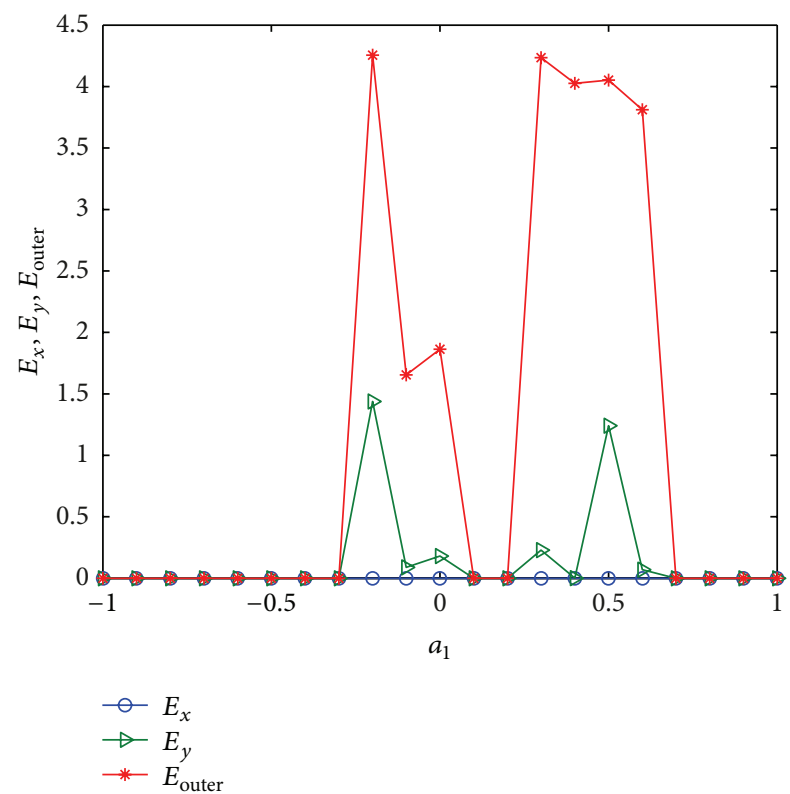

FIgURE 3: The curves of $E_{x}, E_{y}$, and $E_{\text {outer }}$ at $t=400$ concerning $a_{1}$ with $b_{1}=0.2, a_{2}=0.5$, and $b_{2}=0.3$ and $N=10, m_{x}=0.2$, and $m_{y}=0.3$.

synchronization. We consider the following coupled discretetime networks, which are in the form of (1):

$$
\begin{aligned}
x_{i 1}(t+1)= & 1+x_{i 2}(t)-a_{1} x_{i 1}(t)^{2} \\
& +m_{x} \sum_{j=1}^{N} a_{i j}\left(y_{j 1}(t)-x_{j 1}(t)\right),
\end{aligned}
$$

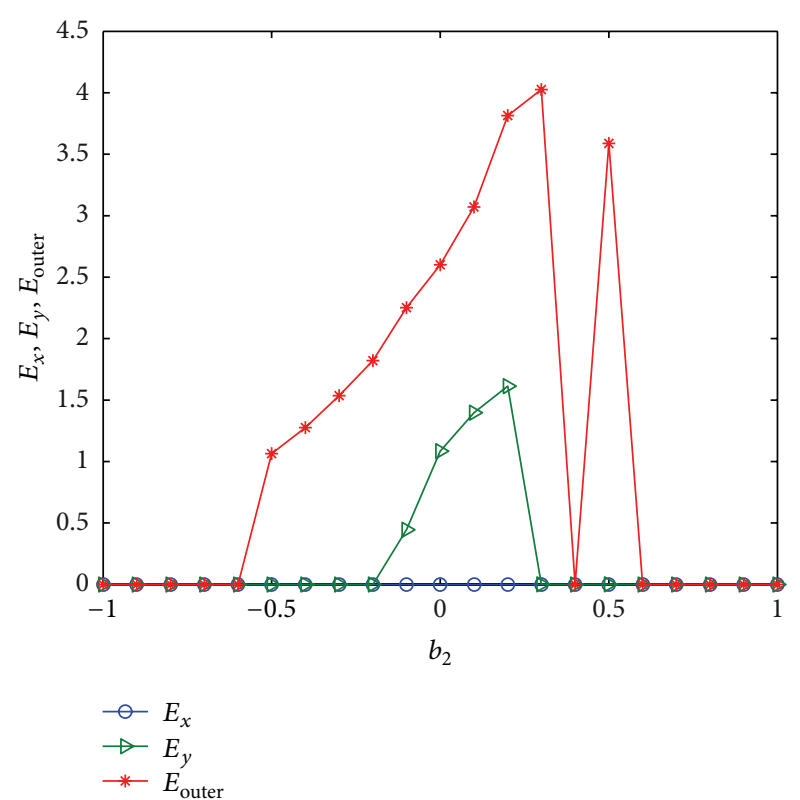

FIGURE 4: The trajectories of $E_{x}, E_{y}$, and $E_{\text {outer }}$ at $t=400$ regarding $b_{2}$ with $a_{1}=0.3, b_{1}=0.2$, and $a_{2}=0.5$ and $N=10, m_{x}=0.2$, and $m_{y}=0.3$.

$$
\begin{aligned}
x_{i 2}(t+1)= & b_{1} x_{i 1}(t) \\
& +m_{x} \sum_{j=1}^{N} a_{i j}\left(y_{j 2}(t)-x_{j 2}(t)\right), \quad i=1,2, \ldots, N, \\
y_{i 1}(t+1)= & 1+y_{i 2}(t)-a_{2} y_{i 1}(t)^{2} \\
& +m_{y} \sum_{j=1}^{N} b_{i j}\left(x_{j 1}(t)-y_{j 1}(t)\right), \\
y_{i 2}(t+1)= & b_{2} y_{i 1}(t)+m_{y} \sum_{j=1}^{N} b_{i j}\left(x_{j 2}(t)-y_{j 2}(t)\right), \\
& i=1,2, \ldots, N,
\end{aligned}
$$

where the node equations in (19) are both Henön maps, which have colorful dynamical properties, for instance, $a_{1}=0.5$ and $b_{1}=0.3$; it has a periodic solution. Since the sum of each row of mutual matrices is one, for simplicity, we take $a_{i j}=b_{i j}=1 / N$ for $i, j=1, \ldots, N$. To measure the extent to which inner synchronization is achieved, we introduce two quantities, $E_{x}=\left\|x_{i}(t)-x_{s}(t)\right\|$ and $E_{y}=\| y_{i}(t)-$ $y_{s}(t) \|, i=1, \ldots, N$. In addition, we denote another quantity $E_{\text {outer }}=\left\|x_{i}(t)-y_{i}(t)\right\|$ for $i=1, \ldots, N$ to demonstrate whether outer synchronization happens. Given the values of $a_{1}=0.3, b_{1}=0.2, a_{2}=0.5$, and $b_{2}=0.3$, we first study the effect of coupling strengths $m_{x}$ and $m_{y}$ on the inner and outer synchronization. Figure 1 shows that the outer synchronization does not happen when the coupling strength is $m_{x}<0.5$, while the inner synchronization inside network $X$ always appears. In the same way, considering the effect of coupling strength $m_{y}$, the details are shown in Figure 2. 


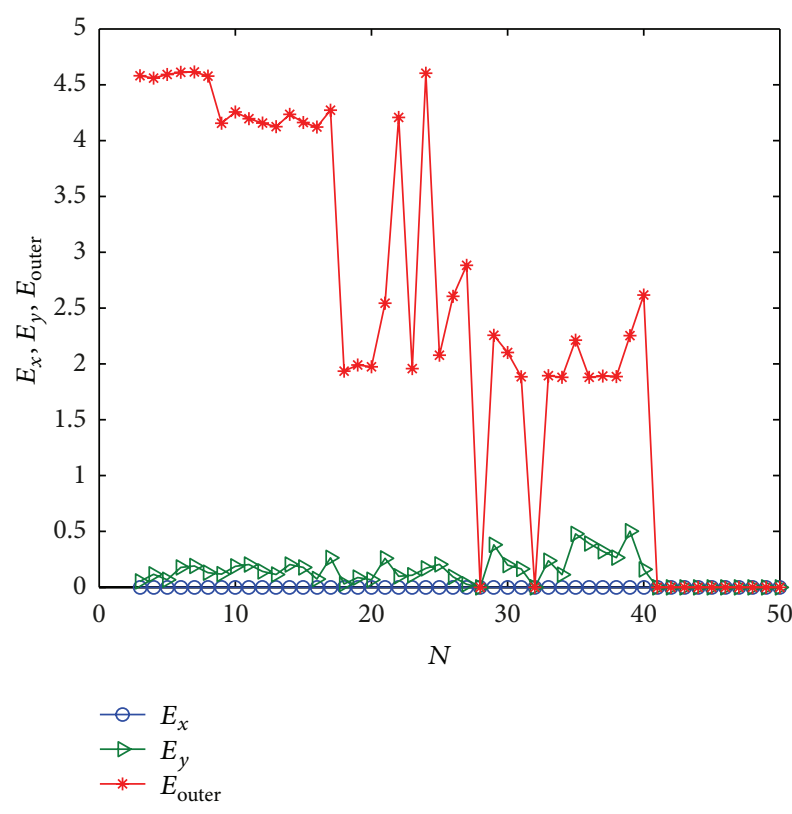

Figure 5: The plots show $E_{x}, E_{y}$, and $E_{\text {outer }}$ at $t=400$ on $N$ with $a_{1}=0.3, b_{1}=0.2, a_{2}=0.5$, and $b_{2}=0.3$ and $m_{x}=0.2$ and $m_{y}=0.3$. The topologies are $a_{i j}=b_{i j}=1 / N, i, j=1, \ldots, N$. Obviously the inner synchronization inside network $Y$ and outer synchronization do not happen for the network size $N<40$ except some values of N.

Next, we discuss the effect of node dynamics on the inner and outer synchronization and take $b_{1}=0.2, a_{2}=0.5$, and $b_{2}=0.3$ and $N=10, m_{x}=0.2$, and $m_{y}=0.3$. We then investigate the effect of parameter $a_{1}$ on the inner and outer synchronization. Similarly, given $a_{1}=0.3, b_{1}=$ 0.2 , and $a_{2}=0.5$ and $N=10, m_{x}=0.2$, and $m_{y}=$ 0.3 , we study the influence of $b_{2}$. The numerical simulations are summarized in Figures 3 and 4, showing that the inner synchronization inside network $X$ always happens, while the inner synchronization inside network $Y$ and the outer synchronization only appear for some values of $a_{1}$ or $b_{2}$.

Finally, we discuss the effect of network size $N$ on the inner and outer synchronization with $a_{i j}=b_{i j}=1 / N, i, j=$ $1, \ldots, N$. Taking the values of $a_{1}=0.3, b_{1}=0.2, a_{2}=0.5$, and $b_{2}=0.3$ and $m_{x}=0.2$ and $m_{y}=0.3$, we plot the curves of $E_{x}, E_{y}$, and $E_{\text {outer }}$ in Figure 5. In the following, we change the topological structures of mutual coupling matrices and choose $A=B$ as a random matrix; the numerics are shown in Figure 6. It is found that the globally connected and random topological structures have similar effect on the inner and outer synchronization. It is noted that the inner synchronization inside network $X$ always happens. A possible reason is the effect of node dynamics. Furthermore, when the Henön map behaves chaotically, no synchronization appears.

\section{Conclusions}

The current study investigated the synchronization between two discrete-time networks with mutual couplings and mainly studied inner synchronization inside each network

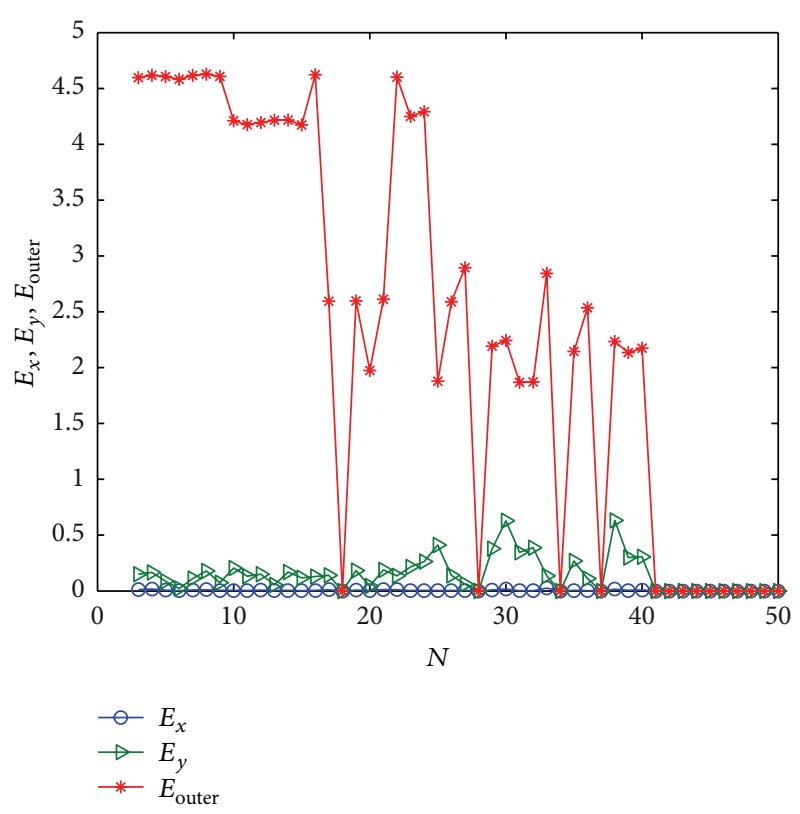

FIgURE 6: The curves of $E_{x}, E_{y}$, and $E_{\text {outer }}$ at $t=400$ regarding $N$ with $a_{1}=0.3, b_{1}=0.2, a_{2}=0.5$, and $b_{2}=0.3$ and $m_{x}=0.2$, and $m_{y}=0.3$. The coupling matrix $A(B)$ is a random matrix satisfying the sum of each row being one.

and outer synchronization between them. We then obtained a synchronous theorem on the inner synchronization inside each network in terms of linear matrix inequality, for the lack of a criterion on the outer synchronization. When the inner synchronization is achieved inside each network and the synchronized states $x_{s}$ and $y_{s}$ are same for a large time, then the outer synchronization will happen. From the numerical simulations, we see that the inner and outer synchronization simultaneously happen when we adjust the values of coupling strengths and parameters in the node dynamics. The globally connected and random topologies have similar effect on the inner and outer synchronization. In addition, outer synchronization is more difficult to achieve than the inner synchronization, meaning that the outer synchronization needs a strong coupling form. Because of the diversity of coupling forms between two networks, deriving the criteria on the inner and outer synchronization simultaneously is a technical challenge, which would be discussed in the future.

\section{Conflict of Interests}

The authors declare that there is no conflict of interests regarding the publication of this paper.

\section{Acknowledgments}

This work was supported by the National Natural Science Foundation of China (nos. 61203155 and 11171084) and Zhejiang Provincial Natural Science Foundation of China under Grant no. LQ12F03003. 


\section{References}

[1] D. J. Watts and S. H. Strogatz, "Collective dynamics of smallworld networks," Nature, vol. 393, no. 6684, pp. 440-442, 1998.

[2] A.-L. Barabási and R. Albert, "Emergence of scaling in random networks," Science, vol. 286, no. 5439, pp. 509-512, 1999.

[3] S. Boccaletti, V. Latora, Y. Moreno, M. Chavez, and D.-U. Hwang, "Complex networks: structure and dynamics," Physics Reports, vol. 424, no. 4-5, pp. 175-308, 2006.

[4] A. Arenas, A. Díaz-Guilera, J. Kurths, Y. Moreno, and C. Zhou, "Synchronization in complex networks," Physics Reports, vol. 469, no. 3, pp. 93-153, 2008.

[5] Q. Wang, Z. Duan, G. Chen, and Z. Feng, "Synchronization in a class of weighted complex networks with coupling delays," Physica A, vol. 387, no. 22, pp. 5616-5622, 2008.

[6] W. Yu, G. Chen, J. Lü, and J. Kurths, "Synchronization via pinning control on general complex networks," SIAM Journal on Control and Optimization, vol. 51, no. 2, pp. 1395-1416, 2013.

[7] M. Sheikhan, R. Shahnazi, and S. Garoucy, "Synchronization of general chaotic systems using neural controllers with application to secure communication," Neural Computing and Applications, vol. 22, no. 2, pp. 361-373, 2013.

[8] X. Li, "Phase synchronization in complex networks with decayed long-range interactions," Physica D, vol. 223, no. 2, pp. 242-247, 2006.

[9] M. Sun, C. Zeng, and L. Tian, "Linear generalized synchronization between two complex networks," Communications in Nonlinear Science and Numerical Simulation, vol. 15, no. 8, pp. 2162-2167, 2010.

[10] D. H. Ji, S. C. Jeong, J. H. Park, S. M. Lee, and S. C. Won, "Adaptive lag synchronization for uncertain complex dynamical network with delayed coupling," Applied Mathematics and Computation, vol. 218, no. 9, pp. 4872-4880, 2012.

[11] W. L. Lu, B. Liu, and T. Chen, "Cluster synchronization in networks of distinct groups of maps," European Physical Journal $B$, vol. 77, no. 2, pp. 257-264, 2010.

[12] Z. Ma, Z. Liu, and G. Zhang, "A new method to realize cluster synchronization in connected chaotic networks," Chaos, vol. 16, no. 2, Article ID 023103, 2006.

[13] C. Li, W. Sun, and J. Kurths, "Synchronization between two coupled complex networks," Physical Review E, vol. 76, no. 4, Article ID 046204, 2007.

[14] H. Tang, L. Chen, J.-A. Lu, and C. K. Tse, "Adaptive synchronization between two complex networks with nonidentical topological structures," Physica A, vol. 387, no. 22, pp. 56235630, 2008

[15] X. Wu, W. X. Zheng, and J. Zhou, "Generalized outer synchronization between complex dynamical networks," Chaos, vol. 19, no. 1, Article ID 013109, 2009.

[16] G. Wang, J. Cao, and J. Lu, "Outer synchronization between two nonidentical networks with circumstance noise," Physica A, vol. 389, no. 7, pp. 1480-1488, 2010.

[17] Y. Z. Sun and D. H. Zhao, "Effects of noise on the outer synchronization of two unidirectionally coupled complex dynamical networks," Chaos, vol. 22, no. 2, Article ID 023131, 2012.

[18] S. Zheng, S. Wang, G. Dong, and Q. Bi, "Adaptive synchronization of two nonlinearly coupled complex dynamical networks with delayed coupling," Communications in Nonlinear Science and Numerical Simulation, vol. 17, no. 1, pp. 284-291, 2012.

[19] M. M. Asheghan, J. Míguez, M. T. Hamidi-Beheshti, and M. S. Tavazoei, "Robust outer synchronization between two complex networks with fractional order dynamics," Chaos, vol. 21, no. 3, Article ID 033121, 2011.

[20] $\mathrm{Z}$. $\mathrm{Wu}$ and $\mathrm{X}$. Fu, "Outer synchronization between driveresponse networks with nonidentical nodes and unknown parameters," Nonlinear Dynamics, vol. 69, no. 1-2, pp. 685-692, 2012.

[21] X. Wu and H. Lu, "Outer synchronization of uncertain general complex delayed networks with adaptive coupling," Neurocomputing, vol. 82, pp. 157-166, 2012.

[22] Y.-Q. Wu, W.-G. Sun, and S.-S. Li, "Anti-synchronization between coupled networks with two active forms," Communications in Theoretical Physics, vol. 55, no. 5, pp. 835-840, 2011.

[23] F. Sorrentino and E. Ott, "Network synchronization of groups," Physical Review E, vol. 76, no. 5, Article ID 056114, 2007.

[24] W. Sun, J. Zhang, and C. Li, "Synchronization analysis of two coupled complex networks with time delays," Discrete Dynamics in Nature and Society, vol. 2011, Article ID 209321, 12 pages, 2011. 


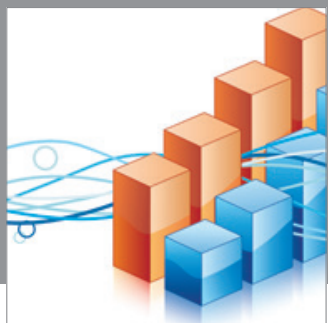

Advances in

Operations Research

mansans

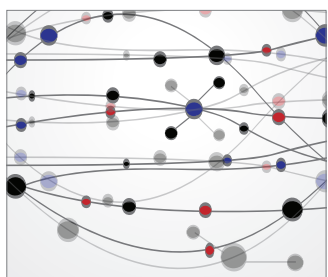

The Scientific World Journal
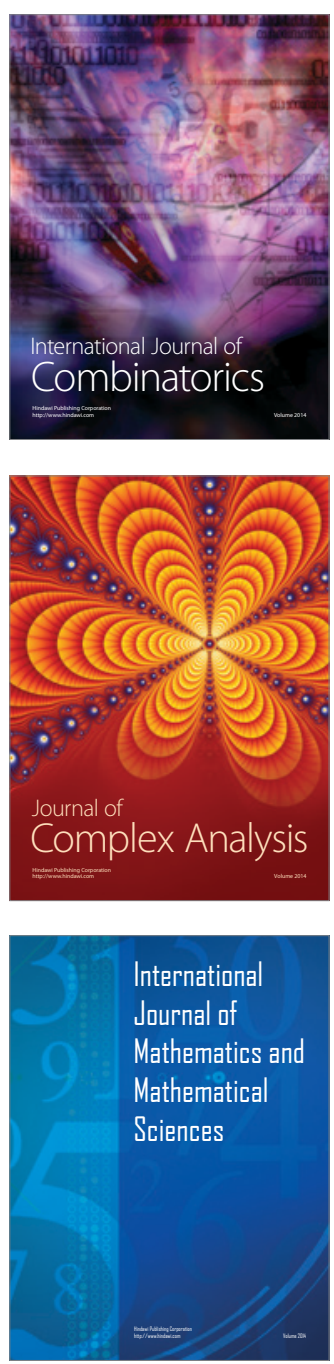
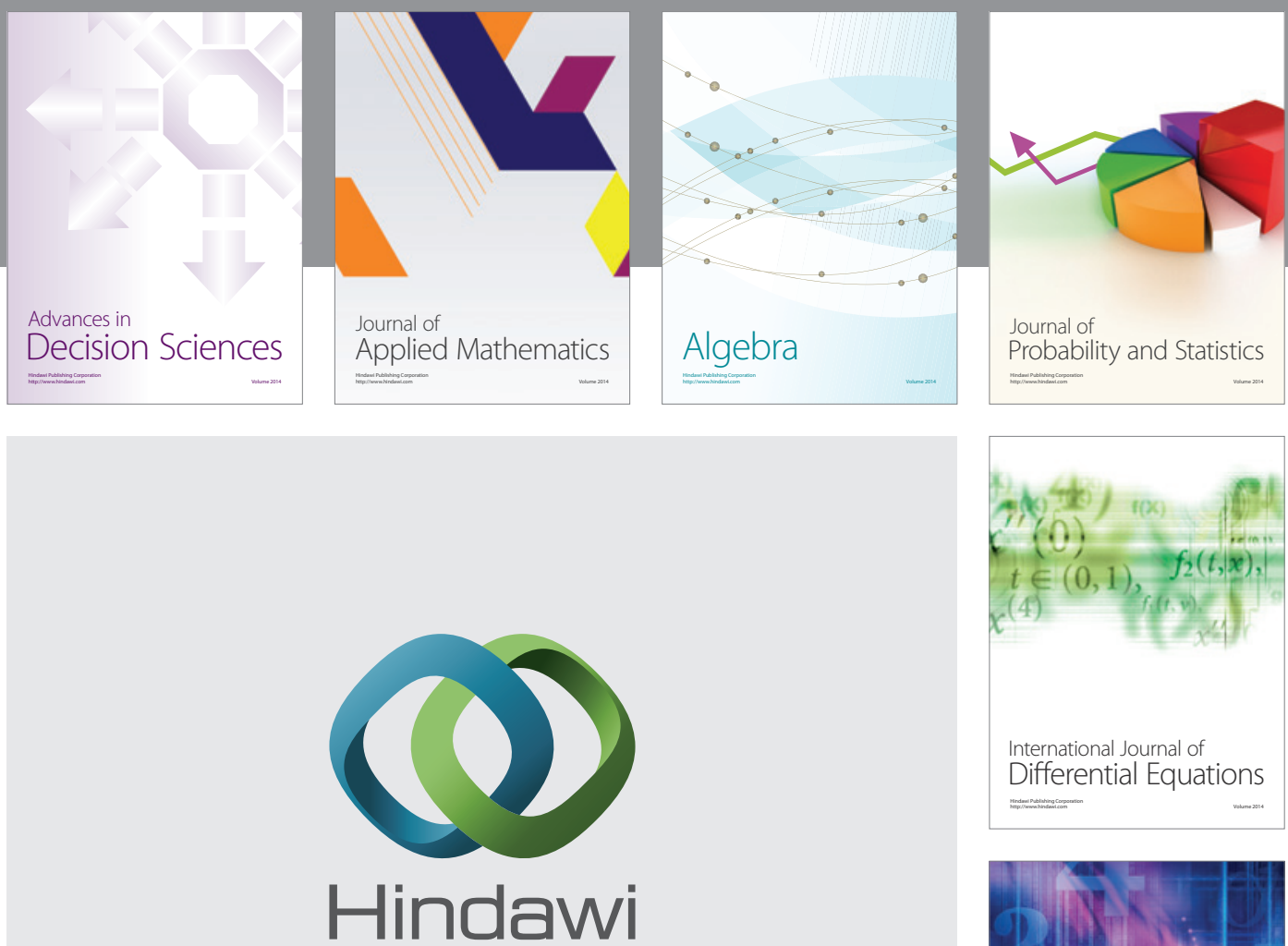

Submit your manuscripts at http://www.hindawi.com
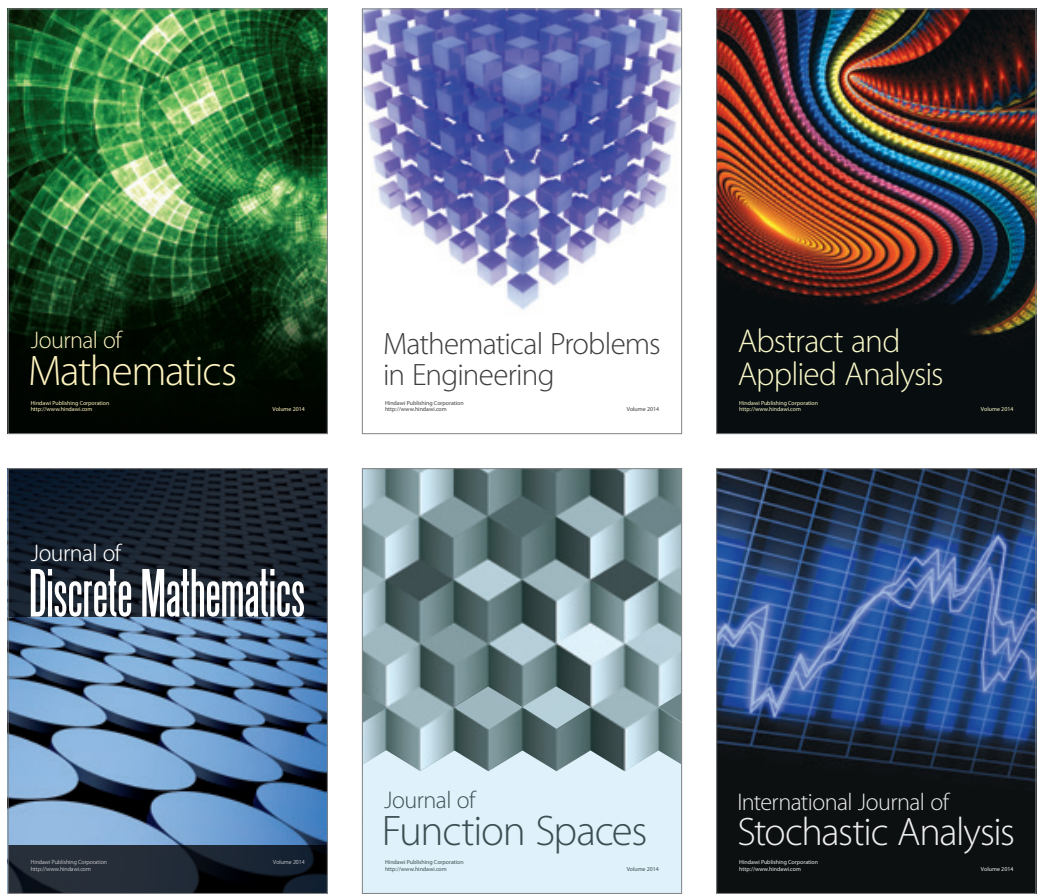

Journal of

Function Spaces

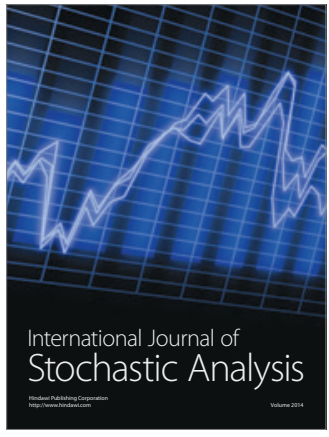

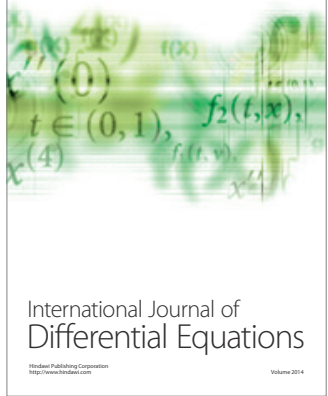
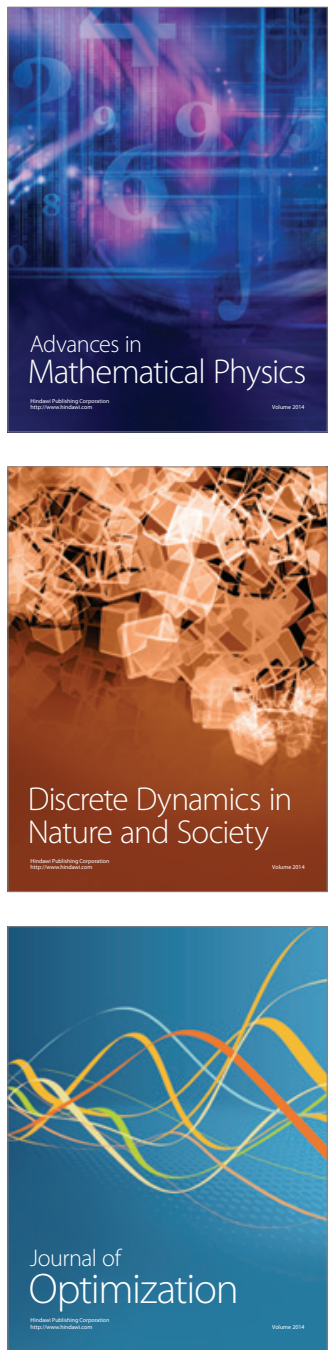